\title{
The Dispersal of an Initial Concentration of Motile Bacteria
}

\author{
By P. C. THONEMANN AND C. J. EVANS \\ Department of Physics, University College of Swansea, Swansea SA2 8PP
}

(Received 30 January 1974; revised 7 May 1975)

SUMMARY

The dispersal of an initial concentration of identical Brownian particles is accurately described by the solution of the conventional diffusion equation, and a diffusion coefficient can be assigned to the assembly of particles. However, the dispersal of an initial concentration of motile bacteria is not well described by the same solution, in spite of the similarity between the random motion of a bacterium and a Brownian particle.

Reasons for the failure of the Gaussian solution of the diffusion equation to describe the dispersal of Escherichia coli are discussed. An equation is formulated which gives the concentration of dispersing organisms as a function of space and time if the speed distribution function of the assembly of organism is known and reproduction is suppressed. For three assumed speed distributions the results are compared with concentrations measured by previous authors.

\section{INTRODUCTION}

When viewed microscopically, the individual organisms in a culture of a motile bacterium, such as Escherichia coli, exhibit a random motion - individual organisms spontaneously change direction in a manner which, superficially, resembles a Brownian particle. Adler \& Dahl (1967) investigated the dispersal of $E$. coli organisms and compared their results with the diffusion of glucose. They measured the rate at which a small, defined concentration of the bacterial population or glucose moved along a capillary tube, and found that, for both, the movement of this 'front' obeyed the relationship $x^{2}=k t$, where $x$ is the distance moved in time $t$ and $k$ is a constant. However, measurement of the concentrations of bacteria and glucose throughout the capillary tube, at a chosen time, showed that it was not possible to describe the concentration of bacteria by the equation which was valid for the diffusion of glucose.

Adler and Dahl used the solution to the diffusion equation given by Ljunggren $\&$ Lamm (1957) as

$$
c(x, t)=\frac{A}{\sqrt{(\pi D t)}} \mathrm{e}^{-x^{2} / 4 D t},
$$

where $c$ is the number of diffusing particles per unit volume, $A$ the number of particles initially distributed uniformly over unit area $x=0$ and $t=0$, and $D$ is a diffusion coefficient.

If $c_{0}=A / \sqrt{ }(\pi D t)$ is the concentration at $x=0, t \neq 0$, then

$$
\ln \left(c / c_{0}\right)=-x^{2} / 4 D t,
$$

and a plot of $\ln \left(c / c_{0}\right)$ against $x^{2}$ gives a straight line with a slope of $-1 / 4 D t$.

Adler \& Dahl's (1967) data for the diffusion of glucose gave straight lines when plotted in this way. However, for bacteria the corresponding plot gave points which did not fall 
on a straight line (Fig. 2). It may be concluded that eqn (I) is not satisfactory for the description of bacterial diffusion.

Adler and Dahl discussed a number of possible reasons for this discrepancy. Amongst these was the suggestion that 'bacteria are heterogeneous with regard to their motility, i.e. some swim faster than others'; this suggestion was not developed further. However, even if the bacteria swim at different speeds, it is not immediately obvious that this would make the diffusion equation invalid, since at any given instant no two Brownian particles are moving at the same speed. The consequences which follow from 'heterogeneous' motility are examined in this paper. We show that a motility (diffusion) coefficient can only be associated with those bacteria which swim at the same speed. The dispersal of a heterogeneous population can then be found by summing over the the motilities of the different speed groups. When a suitable distribution of speeds is assumed, the theoretical curves for the concentration of bacteria along a tube are found to be qualitatively similar to those measured by Adler and Dahl.

\section{A COMPARISON OF THE MOTION OF BACTERIA AND BROWNIAN PARTICLES}

We consider certain properties of the motion of some self-propelled organisms and discuss whether they invalidate the assumptions underlying the derivation of eqn (I).

\section{Energy}

A molecule, or a much larger Brownian particle, is in thermal equilibrium with the medium in which it is immersed, whereas self-propelled bacteria have a much greater average speed of motion than that caused by spontaneous fluctuation within the medium and are not in thermal equilibrium with the medium. In other words, live motile bacteria move faster than dead ones. This feature does not invalidate eqn ( $\mathrm{I}$ ) provided a diffusion coefficient appropriate to a known speed distribution is employed.

\section{Free path}

Berg \& Brown (1972) measured the number of $E$. coli which changed direction in a distance $\delta r$ after having travelled in a straight line of length $r$ from the position of their last change in direction. They found this number decreased exponentially with $r$ and there was no significant correlation between the length of consecutive straight-line paths. However, unlike the changes in the direction of motion of a gas molecule or a Brownian particle because of collisions, the changes in the direction of motion of bacteria are spontaneous, provided the concentration of bacteria is low enough for collisions between bacteria to be ignored. The form of eqn (I) is not altered solely because changes in the direction are spontaneous rather than the result of collisions.

To estimate the upper limit to the concentration for which encounters between bacteria can be neglected, we can assume that an encounter occurs when one bacterium approaches another to within a distance equal to its own dimension. Taking this distance to be $3 \mu \mathrm{m}$ and using the same initial density of bacteria as Adler and Dahl $\left(10^{5}\right.$ to $\left.10^{6} / \mathrm{mm}^{3}\right)$, this gives a mean free path between encounters of 30 to $300 \mu \mathrm{m}$. This distance is between 2 and 20 times the mean free path required to account for the number of spontaneous changes of direction observed by Berg \& Brown (1972) for $E$. coli. Hence encounters between bacteria could have an influence on the rate of dispersal at times close to $t=0$, when concentrations are high, but suich encounters would become relatively rare as dispersal took place. 
It follows that the failure of the usual diffusion equation when the concentration of bacteria is well below the initial value employed by Adler \& Dahl (1967) cannot be explained by collisions between bacteria.

\section{Speed distribution}

It is necessary to distinguish between the time average speed of a single particle (i.e. the average speed of a single particle taken over a considerable period of time) and the average speed characteristic of an assembly of identical particles at a particular time. For the particles of a uniform gas and for an assembly of identical Brownian particles, the average speed of all the particles at a given time is equal to the time average speed of an individual particle. Given a sufficient lapse of time, each particle 'experiences' all the possible speeds present in the assembly at any instant. This is not so for a bacterium. Observation suggests that a bacterium swims so that its speed remains within a narrow range which does not change appreciably after changes in direction. Thus, eqn (I) can be used to describe the dispersal of an assembly of bacteria having speeds which lie within a narrow range but it cannot be used to describe the dispersal of an assembly which possesses a wide range of speeds. Each group of bacteria moving within a narrow speed range possesses a diffusion coefficient which differs from that of another group in a neighbouring speed range. It is this feature of bacterial motion which we propose as an explanation for the failure of eqn (I) to account for the results of Adler and Dahl.

An initial concentration of bacteria will disperse in such a way that bacteria which move at a constant speed greater than the average speed of the assembly will travel further from the starting point than those whose constant speed is less than the assembly average speed. Consequently, the average speed of the bacteria in an element of volume at a given time is an increasing function of the distance of this element of volume from the starting point. Therefore, a diffusion coefficient for an assembly cannot be independent of position and time.

If, after some time has elapsed, the motion of a group of bacteria at a 'front' is followed, then it would be expected that the motion of this front would be described approximately by a diffusion equation, because only bacteria with the highest speeds of motion will be found in this front. This automatic selection of bacteria which swim with speeds in a restricted speed range could account for the observation of Adler and Dahl that eqn (I) is applicable to the motion of a sample at a front.

\section{Initial conditions}

An assumption underlying the derivation of eqn ( $\mathrm{I}$ ) is that there is no correlation between the motion of one particle and any other particle, i.e. each particle proceeds as though no other particles were present.

If it is assumed that all particles are confined to the plane $x=0$ until time $t=0$ and are then released, their initial motion is correlated in the sense that all particles could be assumed to have made a change of direction at time $t=0$. If the probability of changing direction in a distance $\mathrm{d} r$ is independent of the particle's previous history then the release from a plane at time $t=0$ does not invalidate the diffusion equation or its Gaussian solution. In the absence of experimental evidence to the contrary it will be assumed that no error is incurred as a result of this simultaneous release at time $t=0$.

A more serious limitation of the validity of the equation is the necessity to restrict the magnitude of the concentration gradient. The number of particles crossing unit area per second is usually written as

$$
c v=-D \nabla c,
$$




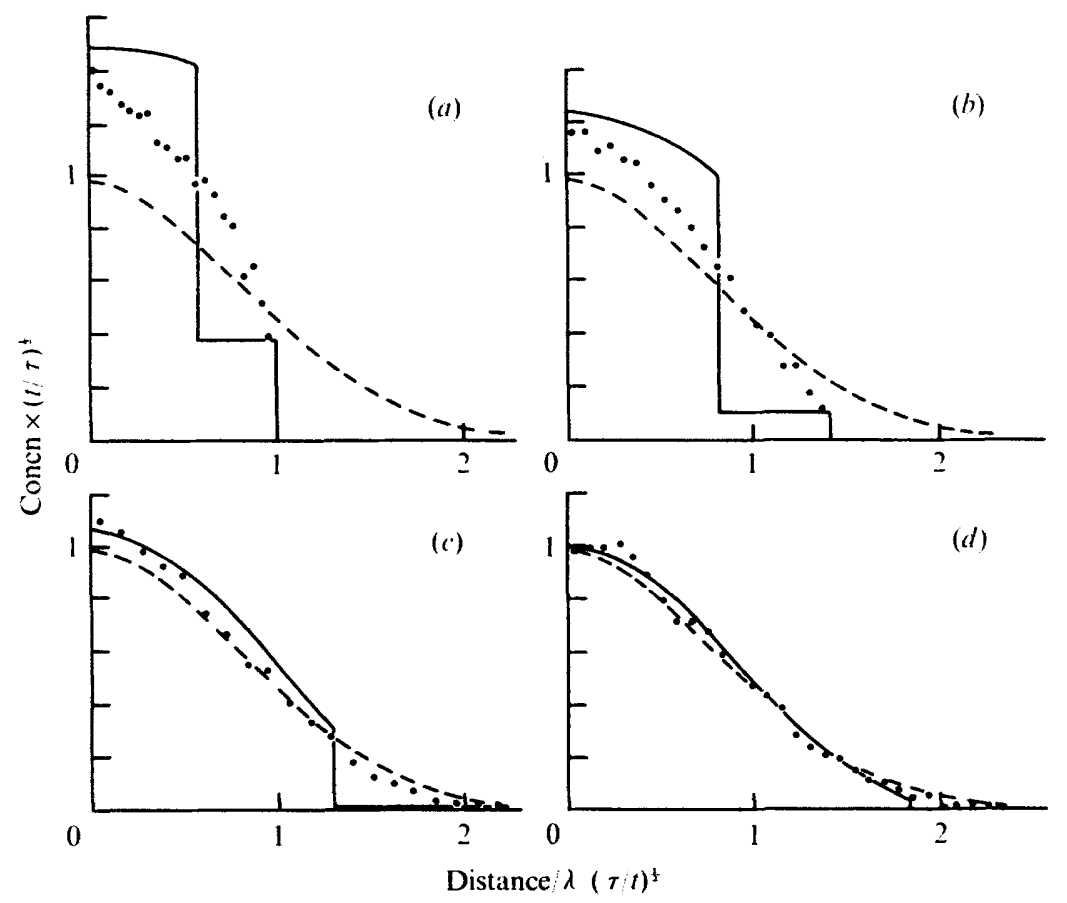

Fig. I. A comparison of the results of three different methods of calculating the concentration of a diffusing substance after release from a plane source. The broken curve is the Gaussian distribution corresponding to eqn ( 1 ). The full curve is the Morse \& Feshbach (1953) distribution modified to preserve the total number of particles. The points are the result of a numerical simulation (which preserves the number of particles). All three methods give the same concentration distribution, within acceptable limits, provided a time of at least $t \geqslant 10 \tau$ has elapsed after release. Values of $t / \tau$ are: (a) $1,(b) 2$, (c) 5 , and (d) 10 .

where $D$ is a diffusion coefficient, $\nabla c$ the concentration gradient and $v$ the average flow velocity. However, the above equation is only valid provided the higher space derivatives of $c$ are neglected (Fowler, 1936). In particular, if $\lambda$ is the mean free path and $\Delta c$ the difference in concentration between two planes separated by a distance $\lambda$

$$
\Delta c=\lambda \frac{\partial c}{\partial x}+\text { higher order derivatives. }
$$

For the higher order derivatives to be neglected it is necessary that

$$
\left|\frac{\lambda}{c} \frac{\partial c}{\partial x}\right| \ll \mathrm{I}
$$

This condition is clearly violated at times close to zero, since $\partial c / \partial x \rightarrow \infty$ as $t \rightarrow 0$.

Another objection to the usual Gaussian solution for describing bacterial dispersal is that it implies that bacteria can reach arbitrarily large distances within a short time of being released. Morse \& Feshbach (1953) have given an alternative diffusion equation, which is intended to avoid this objection, but the solution they give does not preserve the total number of particles. On inspection, it can be seen that the error arises from neglecting the particles which, at time $t$, have not yet made a collision. The missing term can be supplied, but does not remove the difficulty associated with large density gradients.

To clarify the conditions at times close to $t=0$, a numerical calculation of the concentration was carried out using a computer. A Monte-Carlo calculation was made to simulate the 
motion of $10^{4}$ particles from time zero to $10 \tau$, where $\tau$ is the time interval between changes in direction. All particles were assumed to have the same speed, which was constant.

Each particle was started at $x=0$, with its $x$ component of velocity determined by a random number to give a uniform distribution over the interval $-v$ to $v$ for all the particles. The time before change of direction was determined by another number distributed exponentially, with a mean time $\tau$. After each change of direction two more random numbers were chosen, giving the new $x$-component of velocity and the new free time. This process was repeated until the time was Ior. The position of each particle was calculated at $n \tau$ ( $n=\mathrm{I}, 2, \ldots, \mathrm{I} 0$ ), and the numbers, found in the intervals of length $n \lambda / 40$, were stored. From this store the histogram of relative concentration as a function of $x$ was constructed (Fig. I). The standard Gaussian solution (corresponding to eqn I) and that of Morse and Feshback (modified as explained above) are included in Fig. I, for comparison. Neither of the analytical solutions is satisfactory for $t / \tau<2$, but eqn ( $\mathrm{I}$ ) is a valid approximation when $t / \tau>$ I0. Experimentally, it would be diffcult to measure the concentration at $t / \tau \leqslant$ I0 since $E$. coli would only have dispersed an average distance of about $10 \mu \mathrm{m}$ from the plane $x=0$. In practice, it is impossible to release all particles from a plane $x=0$ at time $t=0$, but the dimensions of an initial volume can be made small enough, compared with the distance that some particles have diffused at a later time, for no significant error to be incurred.

These remarks are relevant to the diffusion of Brownian particles as well as to the dispersal of bacteria.

\section{Inhomogeneous population}

If the bacteria are not all identical, then it is necessary to consider first a small group of bacteria for which the diffusion coefficient varies over a small interval from $D$ to $D+\mathrm{d} D$. It is more usual to call this coefficient the motility, $M$ (to distinguish it from the diffusion coefficient of Brownian particles). Let such a group contain $\mathrm{d} N$ bacteria distributed per unit area of the plane $x=0$ at $t=0$. Then the concentration due to this group at times greater than about $10 \tau$ may be written:

$$
\mathrm{d} c(x, t)=\frac{\mathrm{d} N}{\sqrt{(\pi M t)}} \mathrm{e}^{-x^{2} / 4 M t} .
$$

The concentration due to the whole population is found by integrating eqn (2) over all the groups $\mathrm{d} N$, and is conveniently written as:

$$
c(x, t)=\int_{0}^{\infty}(\pi M t)^{-t} \mathrm{e}^{-x^{2} / 4 M t} \frac{\mathrm{d} N}{\mathrm{~d} M} \mathrm{~d} M .
$$

The motility distribution function $\mathrm{d} N / \mathrm{d} M$ is characteristic of the whole population, not just of those bacteria located at $x$ at time $t$. Since there is no known physical basis for calculating $\mathrm{d} N / \mathrm{d} M$ this quantity must be determined experimentally.

To illustrate the particle density distributions which result when eqn '(3) is applicable, we shall make the simplifying assumption that the mean time, $\tau$, between changes of direction is independent of the swimming speed $v$. For one-dimensional dispersal the motility coefficient is $M=v^{2} \tau / 3$.

If $f(v)$ is the speed distribution function, so that $\mathrm{d} N=f(v) \mathrm{d} v$, eqn (3) becomes

$$
c(x, t)=\int_{0}^{\infty}\left(\frac{\pi v^{2} \tau t}{3}\right)^{-t} \mathrm{e}^{-3 x^{2} /<v^{2} \tau t} f(v) \mathrm{d} v
$$

where $N=\int_{0}^{\infty} f(v) \mathrm{d} v$ is the total number of particles distributed over unit area when $x=0$ 


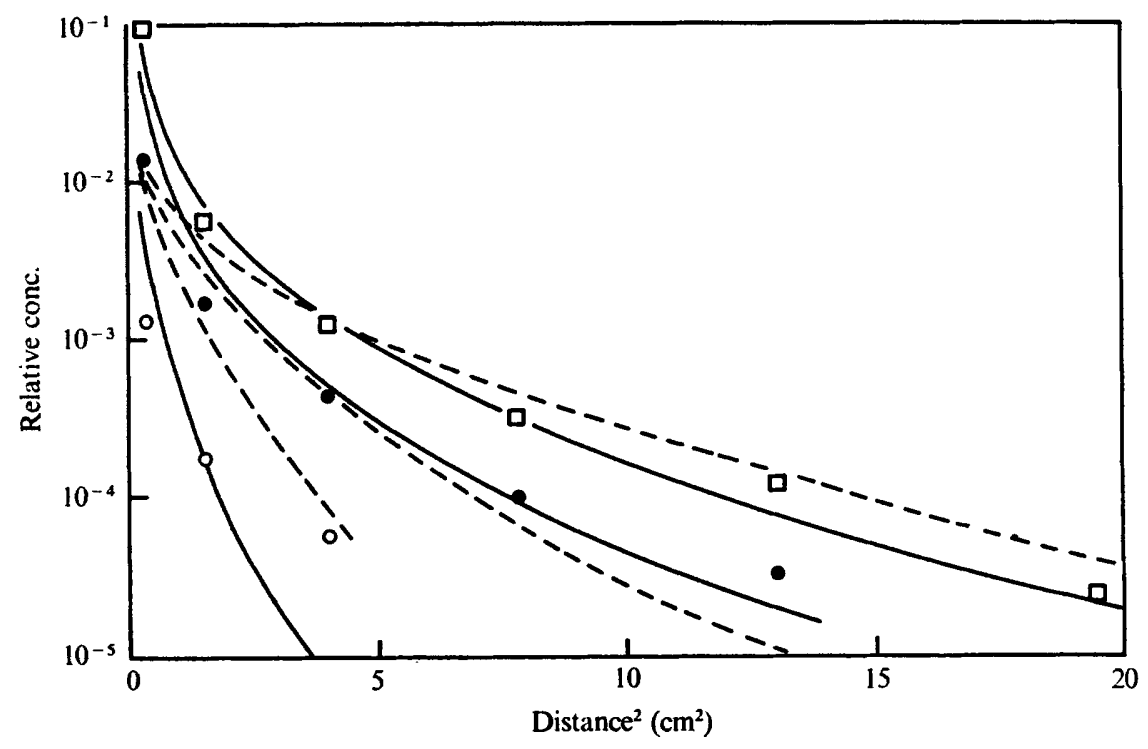

Fig. 2. A comparison of the theoretical and measured concentration of bacteria as a function of position at different times. The dotted and full curves are the theoretical results for a Maxwellian and an exponential distribution of speeds respectively. The points are those given by Adler and Dahl (1967) for E. coli KI 2 strain B275, at (O) I h, (O) $2 \mathrm{~h}$ and ( $\square$ ) $5 \mathrm{~h}$.

and $t=0$. Equation (4) is the proposed solution for $c(x, t)$. To evaluate it, $f(v)$ and $\tau$ must be found by experiment.

To illustrate the difference between the concentration distribution $c(x, t)$ when $M$ is a constant and when $M$ is a function of $v$, three different speed distribution functions will be assumed.

(i) Maxwellian distribution of speeds. The speed distribution function is

$$
f(v)=4 N\left(\frac{k^{3}}{\pi}\right)^{\frac{1}{2}} v^{2} \mathrm{e}^{-k v^{2}}
$$

where $k$ is a constant.

Carrying out the integration of eqn (4) for this $f(v)$ leads to

$$
c(x, t)=\frac{6 N k x}{\pi \tau t} K_{1}\left(\frac{3 k x^{2}}{\tau t}\right)^{\frac{1}{2}},
$$

where $K_{1}$ is a modified Bessel function of the third kind (see, for example, Abramowitz \& Stegun, 1965). Equation (6) is plotted and compared with Adler and Dahl's results in Fig. 2. A value of $12.3 \mathrm{~h} / \mathrm{cm}^{2}$ has been taken for $3 k / \tau$, to obtain a best fit. The agreement with the available experimental data is better than the straight line given by eqn (I).

(ii) Uniform distribution of speeds. In this case $f(v)$ is taken to be constant in the range of $v=0$ to $v=2 V, V$ being the average speed.

Integration of eqn (4) now yields

where

$$
c(x, t)=\frac{N}{2}\left(\frac{3}{\pi \tau t}\right)^{\frac{1}{2}} E_{1}\left(\frac{3 x^{2}}{16 V^{2} \tau t}\right),
$$

$$
E_{1}(z)=\int_{z}^{\infty} \frac{\mathrm{e}^{-8}}{s} \mathrm{~d} s
$$


which is tabulated by Abramowitz \& Stegun (1965). The best fit to the experimental results is found when $3 / 16 V^{2} \tau=0.9 \mathrm{~h} / \mathrm{cm}^{2}$. Equation (7) is not plotted in Fig. 2 but showed slightly better agreement with the experimental points than eqn (6).

(iii) Exponential distribution of speeds. We take $f(v)=N k \mathrm{e}^{-k v} \mathrm{~d} v$, where $k$ is a constant. Equation (4) now yields:

$$
\begin{aligned}
c(x, t) & =N\left(\frac{3}{\pi \tau t}\right)^{\frac{1}{2}} \int_{0}^{\infty} \mathrm{e}^{-1 / u^{2}} \mathrm{e}^{-\alpha u} \frac{\mathrm{d} u}{u} \\
& =N\left(\frac{3}{\pi \tau t}\right)^{t} \mathrm{~F}(\alpha),
\end{aligned}
$$

where $\alpha=\left(3 x^{2} k^{2} / 4 \tau t\right)^{t}, u=k v / \alpha$, and $F(\alpha)$ is the function defined by the integral.

$F(\alpha)$ has been evaluated numerically and a best fit is obtained when $3 k^{2} / 4 \tau=3 \mathrm{I} \mathrm{h} / \mathrm{cm}^{2}$. This result is plotted in Fig. 2 (solid curve) and gives somewhat better agreement with the experimental points than the other two distributions.

\section{CONCLUSIONS}

It is concluded that the simple Gaussian solution to the diffusion equation, valid for the diffusion of identical Brownian particles, cannot be employed to describe the dispersal of motile bacteria because the motion of each bacterium has statistical properties which differ from those of all the other bacteria. As a result, no diffusion coefficient characteristic of an assembly can be defined. However, a dispersal equation can be found which describes the evolution of the particle density with position and time if the motility distribution is known. The resulting dispersal is found to depend on the form of the speed distribution function, as three examples show. It will not be possible to provide a better test of this theory until experimental results are available which give both the speed distribution and more precise data on dispersal for a specific culture of organisms.

We thank Professor P. J. Syrett for many helpful discussions concerning bacterial motion.

\section{REFERENCES}

Abramowitz, M. \& Stegun, I. A. (1965). Handbook of Mathematical Functions. New York: Dover.

ADLER, J. \& DAHL, M. M. (1967). A method for measuring the motility of bacteria and for comparing random and non-random motility. Journal of General Microbiology 46, 16I-173.

BERG, H. C. \& Brown, D. A. (1972). Chemotaxis in Escherichia coli analysed by three-dimensional tracking. Nature, London 239, 500-504.

Fowler, R. H. (1936). Statistical Mechanics, 2nd edn, p. 769. Cambridge: Cambridge University Press.

LJUNGGRen, S. \& LAMm, O. (1957). Diffusion from a bottom layer; diffusion with moving boundaries. Acta chemica scandinavica Ir, 340-359.

Morse, P. M. \& Feshbach, H. (1953). Methods of Theoretical Physics, Part I, p. 865 and Part II, p. 1592. New York: McGraw-Hill. 